\title{
Comparison of GRACE and TIMI risk scores in the prediction of in-hospital and long-term outcomes among East Asian non-ST-elevation myocardial infarction patients
}

Lu Yanqiao ${ }^{1,2 \dagger}$, Lan Shen ${ }^{1,2+}$, Miao Yutong ${ }^{1}$, Shen Linghong ${ }^{1}$ and He Ben ${ }^{1,2^{*}}$

\begin{abstract}
Background: Risk stratification in non-ST segment elevation myocardial infarction (NSTEMI) determines the intervention time. Limited study compared two risk scores, the Thrombolysis in Myocardial Infarction (TIMI) and Global Registry of Acute Coronary Events (GRACE) risk scores in the current East Asian NSTEMI patients.

Methods: This retrospective observational study consecutively collected patients in a large academic hospital between 01/01 and 11/01/2017 and followed for 4 years. Patients were scored by TIMI and GRACE scores on hospital admission. In-hospital endpoints were defined as the in-hospital composite event, including mortality, re-infarction, heart failure, stroke, cardiac shock, or resuscitation. Long-term outcomes were all-cause mortality and cardiac mortality in 4-year follow-up.
\end{abstract}

Results: A total of 232 patients were included (female 29.7\%, median age 67 years), with a median follow-up of 3.7 years. GRACE score grouped most patients (45.7\%) into high risk, while TIMI grouped the majority (61.2\%) into medium risk. Further subgrouping the TIMI medium group showed that half (53.5\%) of the TIMI medium risk population was GRACE high risk ( $\geq 140$ ). Compared to TIMI medium group + GRACE $<140$ subgroup, the TIMI medium + GRACE high-risk ( $\geq 140$ ) subgroup had a significantly higher in-hospital events (39.5\% vs. 9.1\%, $p<0.05)$, long-term all-cause mortality $(22.2 \%$ vs. $0 \% p<0.001)$ and cardiac death $(11.1 \%$ vs. $0 \% p=0.045)$ in 4-year follow-up. GRACE risk scores showed a better predictive ability than TIMl risk scores both for in-hospital and long-term outcomes. (AUC of GRACE vs. TIMI, In-hospital: 0.82 vs. 0.62 ; long-term mortality: 0.89 vs. 0.68 ; long-term cardiac mortality: 0.91 vs. 0.67 , all $p<0.05)$. Combined use of the two risk scores reserved both the convenience of scoring and the predictive accuracy.

Conclusion: GRACE showed better predictive accuracy than TIMI in East Asian NSTEMI patients in both in-hospital and long-term outcomes. The sequential use of TIMI and GRACE scores provide an easy and promising discriminative tool in predicting outcomes in NSTEMI East Asian patients.

Keywords: NSTEMI, GRACE, TIMI, Outcome assessment

*Correspondence: drheben@126.com

† Lu Yanqiao and Lan Shen contributed equally to the study.

1 Department of Cardiology, Shanghai Chest Hospital, Shanghai Jiao Tong University, Shanghai, China

Full list of author information is available at the end of the article

\section{Background}

Cardiovascular disease remains the main contributor to the cause of death globally $[1,2]$. The prevalence of acute coronary syndromes (ACS) has increased significantly during the recent 30 years in China $[2,3]$. A major 
component of ACS, Non-ST segment elevation myocardial infarction (NSTEMI), has more than twice the incidence compared to ST-segment elevation myocardial infarction (STEMI) $[4,5]$. However, NSTEMI patients have a large range of clinical consequences, from minimal sequelae to early death [6]. Risk assessment was crucial in guiding therapeutic decision-making. Guidelines recommend that high-risk patients receive more aggressive invasive treatment upon risk stratification on admission [7-9]. Therefore, there is an essential need to assess individual risk easily and accurately. GRACE and TIMI are the two most popular prediction models in ACS developed during the prereperfusion era and have been compared face to face mainly in the Caucasian population $[10,11]$. Studies proved TIMI's advantage in its simplicity in clinical application and GRACE's favorable discriminative power $[12,13]$. Nevertheless, limited evidence compared the usefulness of these two major risk scores and their association with both in-hospital events and longterm outcomes in contemporary East Asian patients.

In this observational study, we sought to (1) examine the distribution of risk tertiles by GRACE versus TIMI in a contemporary cohort of NSTEMI East Asian patients in today's reperfusion era, (2) compare the predictive ability between GRACE and TIMI risk score, (3) explore the optimal way for risk stratification in East Asian population by combining these two scores.

\section{Methods}

\section{Study population}

The study population began with 1659 patients consecutively hospitalized with primary diagnose as ACS in the cardiovascular department in Shanghai Renji hospital, China, from Jan 1st, 2017 to Nov 1st, 2017. Participants were at least 18 years old, admitted to the hospital with a presumptive ACS diagnosis according to the following points: clinical symptoms, typical ischemic ECG changes, elevated cardiac biomarkers, and documented prior coronary artery diseases [14]. Participants diagnosed with STEMI were excluded. Patients were also excluded if the symptom was caused by severe trauma, medical surgery, or other serious comorbidities. Briefly, we included NSTEMI patients with acute chest discomfort, elevation of cardiomyocyte necrosis biomarkers, ECG characteristics (transient ST-segment elevation, persistent or transient ST-segment depression, flat or inverted $\mathrm{T}$ waves, pseudo-normalization of T waves, or normal ECG) [14].

\section{Risk scores}

Both GRACE and TIMI risk scores were calculated on admission. In UA/NSTEMI, TIMI scores incorporated the following 7 points: age $\geq 65$ years, $\geq$ three coronary artery disease (CAD) risk factors, known coronary stenosis $>50 \%$, aspirin used in the past 7 days, severe angina ( $\geq 2$ episodes w/in $24 \mathrm{~h}$ ), severe angina ( $\geq 2$ episodes $\mathrm{w} / \mathrm{in} 24 \mathrm{~h}$ ) and positive cardiac marker. Patients achieved 1 point for yes and 0 points for no. Patients with 0-2 scores were low-risk groups while 3-4 scores were medium-risk groups and 5-7 scores were high-risk.

GRACE score was calculated on admission based on the following variables: age, heart rate (HR), systolic blood pressure (SBP), initial serum creatine (Cr), Killip classification, cardiac arrest at presentation, initial cardiac enzyme positive, and ST-segment deviation. Patients who scored $\leq 108$ points were low-risk, $109-139$ points were medium-risk, $\geq 140$ points were high-risk. We also used online risk calculators following the recommendation by 2020 ESC guideline, https://www.outcomesumassmed.org/risk_models_grace_orig.aspx [14].

To explore the optimal risk score in our study population, we examine a way to reserve the two risk scores' convenience and predictive accuracy. We tested the method to use the combined score, which further stratified TIMI medium-risk patients into the GRACE $\geq 140$ subgroup and GRACE $<140$ subgroups.

\section{Outcomes}

The primary outcomes of interest for the present study were the following: (1) in-hospital composite endpoint including all-cause mortality, re-infarction, heart failure, cardiac shock, resuscitation, and in-hospital stroke, (2) long-term outcomes were all-cause mortality and cardiac mortality from discharge to 4-year follow-up postdischarge. Long-term all-cause death information was collected through two independent clinical follow-up fellows by telephone or email.

\section{Statistical analysis}

Continuous variables were presented using medians with interquartile ranges (IQRs) and were compared using Wilcoxon rank-sum tests. Categorical variables were presented as percentages and compared using MantelHaenszel $X^{2}$ tests. The accuracy of predicting in-hospital and long-term outcomes between TIMI and GRACE score was evaluated by the Receiver Operating Characteristic Curve (ROC) curves. Discriminatory performance was measured by the c-statistic (area under the receiver-operating characteristic curve) comparing the TIMI risk score with the GRACE risk score. Kappa value was calculated to analyze the consistency of TIMI and GRACE risk scores. Kappa value $<0.4$ was considered an inconsistency between the two comparisons. SPSS version 21.0 (IBM) was used to analyze all the statistical data, and a two-sided $p$ value $<0.05$ was considered statistically significant. 


\section{Results}

Of 1659 ACS patients screened for the study, 261 patients were hospitalized as NSTEMI with positive troponin test. Among these NSTEMI patients, 29 participants were excluded with missing clinical data, leaving 232 participants were eligible as NSTEMI patients in our study (Fig. 1).

Among the NSTEMI patients, $163(70.3 \%)$ patients were male, while $69(29.7 \%)$ patients were female. In the 232 patients, 224 (96.6\%) underwent coronary angiography, and $166(74.1 \%)$ patients underwent revascularization. More than half (65.9\%) of patients suffered from hypertension, $32.8 \%$ had a history of diabetes, $5.2 \%$ had hyperlipidemia, and $38.8 \%$ had a smoking history. One in five had a history of myocardial infarction, and $18.1 \%$ had prior PCI history (Table 1).

TIMI and GRACE risk scores were evaluated for every patient on admission. The median TIMI risk score was 3.0 (2.0-4.0), and the median GRACE score was 137.0 (114.3-157.8). These 232 patients were divided into three groups (high-risk group, medium-risk group, and low-risk group) based on their risk scores. There was a large discordance between these two risk scores. GRACE grouped most people into high-risk (45.7\%) while TIMI grouped the majority into medium-risk (61.2\%) (Additional file 1: Table S1). Half of the TIMI medium-risk group (53.5\%) were GRACE high-risk patients. Similarly, the majority of the TIMI low-risk group was GRACE medium-risk patients (43.5\%). In contrast, the majority of GRACE high-risk patients (71.7\%) were TIMI medium risk. There is little correlation between GRACE and

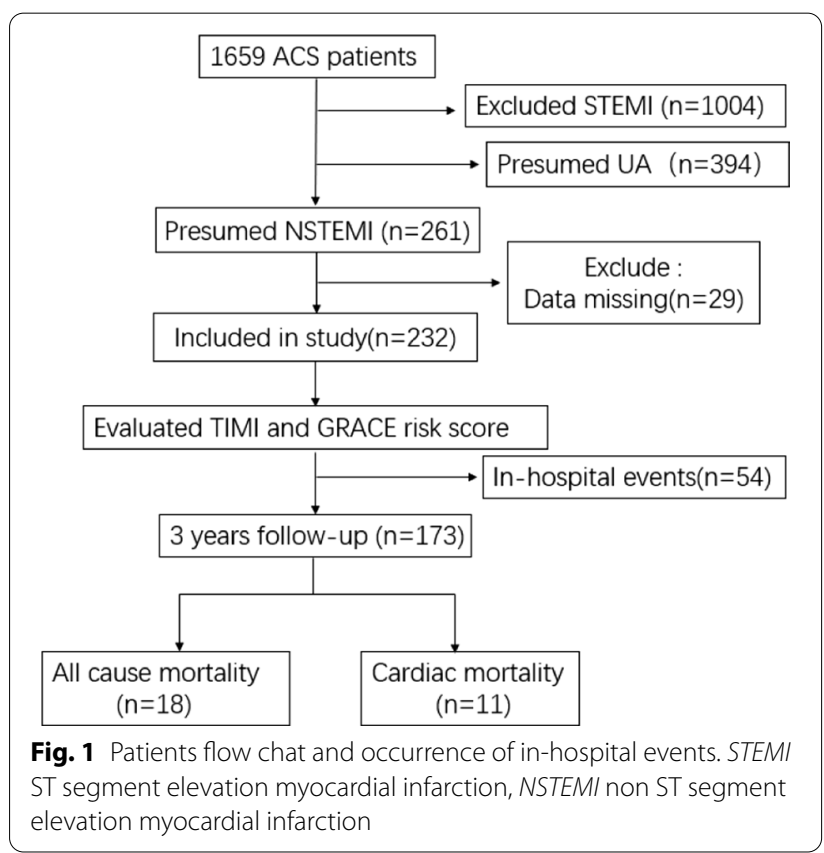

TIMI risk score. (Kappa value $=0.077$, Additional file 1: Table S1 and Fig. 2).

In-hospital events in patients grouped by GRACE score were $38.7 \%$ in GRACE high, $11.8 \%$ in GRACE medium, and $7.3 \%$ in GRACE low-risk groups (Table 2). In-hospital events grouped by TIMI score were $38.1 \%$ in TIMI high, $25.4 \%$ in TIMI medium, and $14.5 \%$ in TIMI low-risk patients (Table 2). Notably, the TIMI medium and GRACE medium showed a significant difference in the incidence of in-hospital events $(25.4 \%$ vs. $11.8 \%$, $p=0.014$, Table 2). When we mixed the medium-risk group with the high-risk group patients, the prediction difference of TIMI and GRACE risk scores disappeared (Additional file 2: Table S2).

We used ROC curves to evaluate the accuracy of predicting in-hospital events with GRACE and TIMI risk scores. As shown in Fig. 3, both GRACE and TIMI risk scores had predictive value for the occurrence of in-hospital events. However, GRACE risk scores showed better predictive ability than TIMI risk scores (C-statistic for GRACE versus TIMI: $0.82,95 \%$ CI $0.75-0.89$ vs. 0.62 , 95\% CI 0.54 to $0.71, p<0.001)$.

During the 4-year follow-up, 59 of them lost to followup. We compared patients who had follow-up and those who lost follow-up. The baseline characteristics were similar between the two groups (Table 1).

During the 4-year follow-up (median follow-up time was 3.7 years, the interquartile range was $3.4-3.9$ years), 18 all-cause deaths were observed, 11 of them were cardiac mortality (Fig. 1). For GRACE grouping, all death occurred in GRACE high-risk group (Table 2). For TIMI grouping, the high, medium, and low-risk group had a mortality rate of $18.8 \%, 11.4 \%$, and $5.8 \%$, respectively (Table 2). The cardiac mortality rate in GRACE high-risk group was $14.5 \%$. In the TIMI high, medium, low-risk group, the cardiac mortality incidence was $18.8 \%, 5.8 \%$, $3.8 \%$. GRACE score showed higher prediction ability than TIMI score in all-cause mortality and cardiac mortality (AUC under ROC curves: 0.89 vs. 0.68, 0.91 vs. 0.67, Fig. 3).

Similarly, the incidence of long-term outcomes was significantly different between TIMI medium-risk group and GRACE medium-risk group (Table 2). However, when we combined the medium and high-risk groups, the predictive difference disappeared between TIMI and GRACE risk scores (Additional file 2: Table S2).

Due to significant heterogeneity in TIMI mediumrisk groups, we further selected out high-risk patients among TIMI medium-risk groups by subsequently using the GRACE score. We first apply the TIMI score for initial screening. If patients were TIMI low or TIMI high, we stopped. If patients were TIMI medium, we used the GRACE score for further subgrouping (Fig. 4). Compared 
Table 1 Baseline characteristics of patients with NSTEMI

\begin{tabular}{|c|c|c|c|c|}
\hline Variables & Patients $(n=232)$ & $\begin{array}{l}\text { With 4-year followup } \\
(n=173)\end{array}$ & $\begin{array}{l}\text { Without 4-year follow-up } \\
(n=59)\end{array}$ & $p$ value* \\
\hline \multicolumn{5}{|l|}{ Admission baseline } \\
\hline Sex (male: female) & 70.3\%: $29.7 \%$ & 71.1\%: $28.9 \%$ & 67.8\%: $32.2 \%$ & 0.743 \\
\hline Age (years) & $67.0(59.0-74.0)$ & $67.0(59.5-74.0)$ & $68.0(58.0-76.0)$ & 0.721 \\
\hline HR (beats/min) & $76.0(70.0-82.0)$ & $75.0(66.3-80.0)$ & $78.0(70.0-82.0)$ & 0.623 \\
\hline $\mathrm{DBP}(\mathrm{mmHg})$ & $77.0(70.0-85.0)$ & $77.0(70.5-85.0)$ & $76.0(70.0-82.0)$ & 0.960 \\
\hline $\mathrm{SBP}(\mathrm{mmHg})$ & 137.5 (125.0-154.0) & $139.0(125.0-154.5)$ & $136.0(125.0-153.0)$ & 0.726 \\
\hline GLU (mmol/L) & $5.6(4.8-7.2)$ & $5.5(4.8-6.6)$ & $5.8(4.9-8.6)$ & 0.163 \\
\hline $\mathrm{Hb}(\mathrm{g} / \mathrm{L})$ & $135.0(120.8-147.3)$ & $136.0(121.3-147.0)$ & $132.0(117.0-148.3)$ & 0.605 \\
\hline $\operatorname{PLT}\left({ }^{*} 10^{9} / \mathrm{L}\right)$ & $202.0(166.5-246.5)$ & $201.0(167.5-242.0)$ & $208.0(163.3-258.8)$ & 0.619 \\
\hline APTT (s) & $27.6(25.7-29.9)$ & $27.5(25.7-29.8)$ & $27.9(25.6-31.5)$ & 0.709 \\
\hline $\mathrm{SCr}(\mu \mathrm{mmol} / \mathrm{L})$ & $77.0(64.9-93.0)$ & $77.0(64.5-92.0)$ & $75.3(64.8-101.1)$ & 0.536 \\
\hline LVEF (\%) & $60.0(55.0-66.0)$ & $61.0(55.0-67.0)$ & $59.5(55.0-64.0)$ & 0.439 \\
\hline LVEDV/BSA (ml) & $47.0(43.0-50.0)$ & $46.5(43.0-51.0)$ & $47.5(42.3-50.0)$ & 0.543 \\
\hline CKMB (IU/L) & $20.3(13.5-34.5)$ & $21.3(13.6-38.1)$ & $18.3(12.8-31.1)$ & 0.272 \\
\hline $\operatorname{Tn}$ I (ng/ml) & $1.0(0.2-5.1)$ & $1.3(0.2-5.7)$ & $0.9(0.2-2.9)$ & 0.384 \\
\hline CK (U/L) & $128.0(79.0-321.5)$ & $134.0(78.0-340.0)$ & $113.0(79.0-305)$ & 0.619 \\
\hline PeakTNI (ng/ml) & $9.45(2.3-18.2)$ & $8.73(3.5-15.3)$ & $10.21(2.2-16.2)$ & 0.732 \\
\hline Peak CK (ng/ml) & $203.0(68.0-340.0)$ & $225.0(89.0-354.0)$ & $194.0(65.0-332.0)$ & 0.630 \\
\hline LDL-C (mmol/L) & $2.6(1.9-3.1)$ & $2.6(1.8-3.1)$ & $2.7(2.0-3.5)$ & 0.276 \\
\hline $\mathrm{HDL}-\mathrm{C}(\mathrm{mmol} / \mathrm{L})$ & $1.0(0.8-1.2)$ & $1.0(0.8-1.2)$ & $1.0(0.8-1.2)$ & 0.743 \\
\hline $\mathrm{TG}(\mathrm{mmol} / \mathrm{L})$ & $1.6(1.1-2.3)$ & $1.5(1.2-2.2)$ & $1.8(1.1-2.4)$ & 0.302 \\
\hline CRP (mg/L) & $3.2(1.0-9.0)$ & $12.8(1.0-8.6)$ & $4.4(1.0-9.5)$ & 0.619 \\
\hline BNP (pg/mL) & $136.0(61.5-327.5)$ & $114.0(58.0-316.0)$ & $146.5(89.6-345.0)$ & 0.131 \\
\hline \multicolumn{5}{|l|}{ Previous disease (\%) } \\
\hline Hypertension & 65.9 & 67.6 & 61.0 & 0.427 \\
\hline Hyperlipidemia & 5.2 & 5.2 & 5.1 & 1.000 \\
\hline Diabetes & 32.8 & 30.1 & 40.7 & 0.150 \\
\hline Smoking & 38.8 & 38.7 & 39.0 & 1.000 \\
\hline Ischemia stroke & 13.4 & 11.0 & 20.3 & 0.068 \\
\hline Renal insufficiency & 8.6 & 9.2 & 6.8 & 0.609 \\
\hline Angina & 40.9 & 42.2 & 37.3 & 0.543 \\
\hline Prior heart failure & 3.9 & 4.0 & 3.4 & 1.000 \\
\hline Prior Ml & 19.0 & 18.5 & 20.3 & 0.848 \\
\hline $\mathrm{PCl}$ & 18.1 & 16.8 & 22.0 & 0.433 \\
\hline $\begin{array}{l}\text { CABG } \\
\text { Killip classes }\end{array}$ & 2.6 & 3.5 & 0 & 0.342 \\
\hline 1 & 79.3 & 79.2 & 79.7 & 0.939 \\
\hline$\|$ & 4.7 & 4.6 & 5.1 & 1.000 \\
\hline III & 9.5 & 9.2 & 10.2 & 0.835 \\
\hline IV & 6.5 & 6.9 & 5.1 & 0.847 \\
\hline Cardiac arrest at admission & 0.4 & - & - & - \\
\hline \multicolumn{5}{|l|}{ Discharge medication (\%) } \\
\hline Statins & 91.8 & 90.2 & 96.6 & 0.200 \\
\hline ACE inhibitors or ARBs & 46.1 & 46.8 & 44.1 & 0.714 \\
\hline$\beta$-blockers & 64.2 & 67.6 & 54.2 & 0.064 \\
\hline \multicolumn{5}{|l|}{ Riskscores } \\
\hline GRACE scores & 137.0 (114.3-157.8) & $137.0(114.5-157.0)$ & $140.0(113.0-164.0)$ & 0.764 \\
\hline TIMI scores & $3.0(2.0-4.0)$ & $3.0(2.0-4.0)$ & $3.0(2.0-4.0)$ & 0.564 \\
\hline
\end{tabular}


Table 1 (continued)

Values are showed as median (interquartile range), or percentage (n\%)

$H R$ heart rate, DBP diastolic blood pressure, SBP systolic blood pressure, GLU blood glucose, $T G$ triglyceride, $H b$ hemoglobin, $P L T$ platelet, $A P T T$ activated partial thromboplastin time, CKMB creatine phosphokinase-Mb, SCr serum creatinine, CK creatine kinase, CRP C-reactive protein, BNP brain natriuretic peptide, LEVF left ventricular ejection fraction, LVEDV/BSA left ventricular end-diastolic volume/body surface area, $M I$ myocardial infarction

${ }^{*} p<0.05$
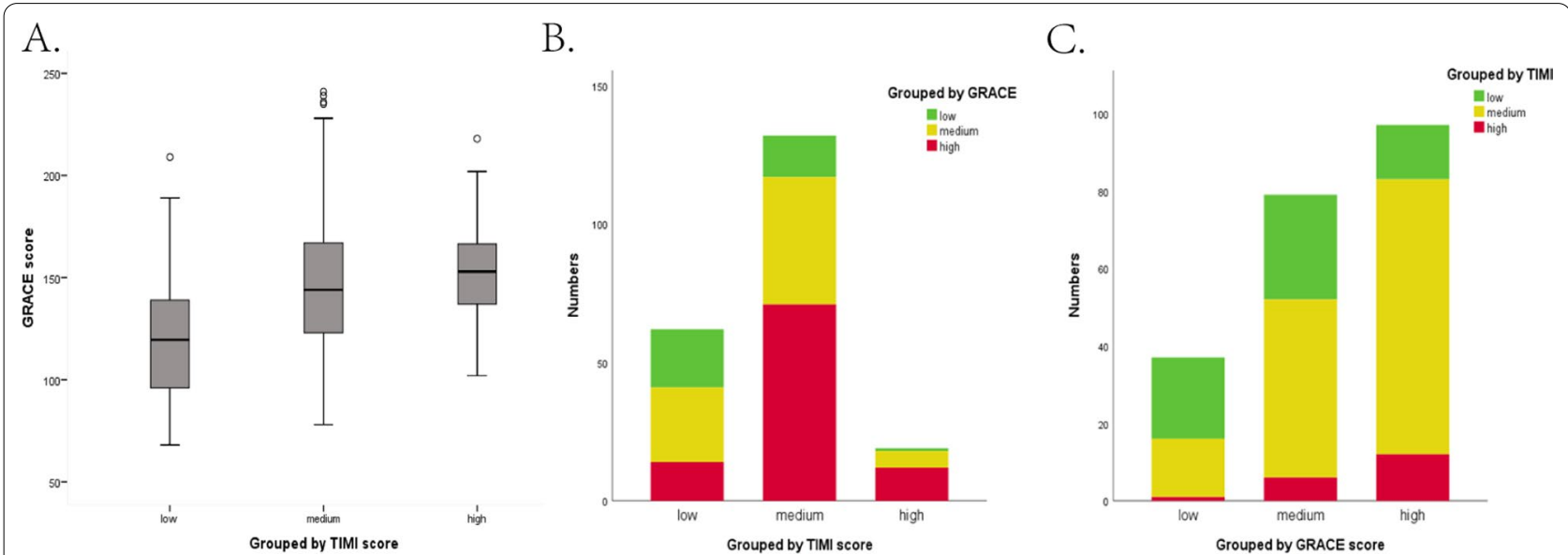

Fig. 2 The correlation between TIMI and GRACE risk scores showed on the scatterplot and boxplot. A The boxplot showed the mean GRACE score ( $Y$ axis) in different TIMI risk groups ( $X$ axis). B The bar graph showed the proportion of different GRACE risk grades ( $Y$ axis) in different TIMI risk groups ( $X$ axis). C The bar graph showed the proportion of different TIMl risk grades ( $Y$ axis) in different GRACE risk groups ( $X$ axis)

Table 2 Occurrence of in-hospital events and long-term outcomes in patients grouped by TIMI and GRACE risk scores

\begin{tabular}{llll}
\hline & GRACE risk score & TIMI risk score & $\boldsymbol{p}$ value \\
\hline $\begin{array}{llll}\text { In-hospital events } \\
\text { Low risk }\end{array}$ & $3(41)$ & & \\
Medium risk & $10(85)$ & $10(69)$ & 0.411 \\
$\begin{array}{l}\text { High risk } \\
\text { All-cause mortality }\end{array}$ & $41(106)$ & $36(142)$ & $0.014^{*}$ \\
Low risk & $0(29)$ & $8(21)$ & 0.960 \\
Medium risk & $0(68)$ & $3(52)$ & \\
High risk & $18(76)$ & $12(105)$ & 0.549 \\
Cardiac mortality & & $3(16)$ & $0.01^{*}$ \\
Low risk & $0(29)$ & & 0.921 \\
Medium risk & $0(67)$ & $2(52)$ & 0.535 \\
High risk & $11(76)$ & $6(104)$ & 0.082 \\
\hline
\end{tabular}

Results are showed as value $(n) . * 0<0.05$

with the TIMI medium + GRACE high risk $(<140)$ subgroup, patients in TIMI medium + GRACE $\geq 140$ subgroup were older, had higher brain natriuretic peptide (BNP), and were more likely to have comorbidities such as a history of stroke and heart failure. (Additional file 3: Table S3) Among the TIMI medium group, the GRACE $\geq 140$ subgroup had an almost six-fold increased risk of in-hospital death than the GRACE $<140$ subgroup (39.5\% vs. $9.1 \%$, odds ratio: $6.52,95 \%$ CI $2.5-17.0$, $p<0.001)$.

Among the 142 TIMI medium-risk patients, 76 were sub-grouped as GRACE high ( $\geq 140$ ), and 54 were followed up for 4 years. (Table 3) Similar to in-hospital events, the TIMI medium + GRACE high $(\geq 140)$ subgroup had higher all-cause mortality and cardiac death. (all-cause mortality: $22.2 \%$ vs. $0 \% p<0.001$, cardiac death: $11.1 \%$ vs. $0 \% p=0.045$, Table 3 ). In short, TIMI medium-risk group had a widely divergent trend in event risk. The TIMI medium + GRACE high $(\geq 140)$ subgroup has a similar event risk to the TIMI high-risk group, whereas the TIMI medium + GRACE $<140$ subgroup has a similar event risk to the TIMI low-risk group.

\section{Discussion}

In today's reperfusion era, risk score decides the optimal timing of intervention among NSTEMI, yet the optimal invasive time and specific long-term management by risk stratification are still upon disputation [15-17]. Moreover, the significant difference in patient characteristics between Caucasian and Asian NSTEMI patients further highlights the need to examine TIMI and GRACE risk scores in East Asian NSTEMI patients [18]. To accurately assess the discriminative power of GRACE and TIMI risk 


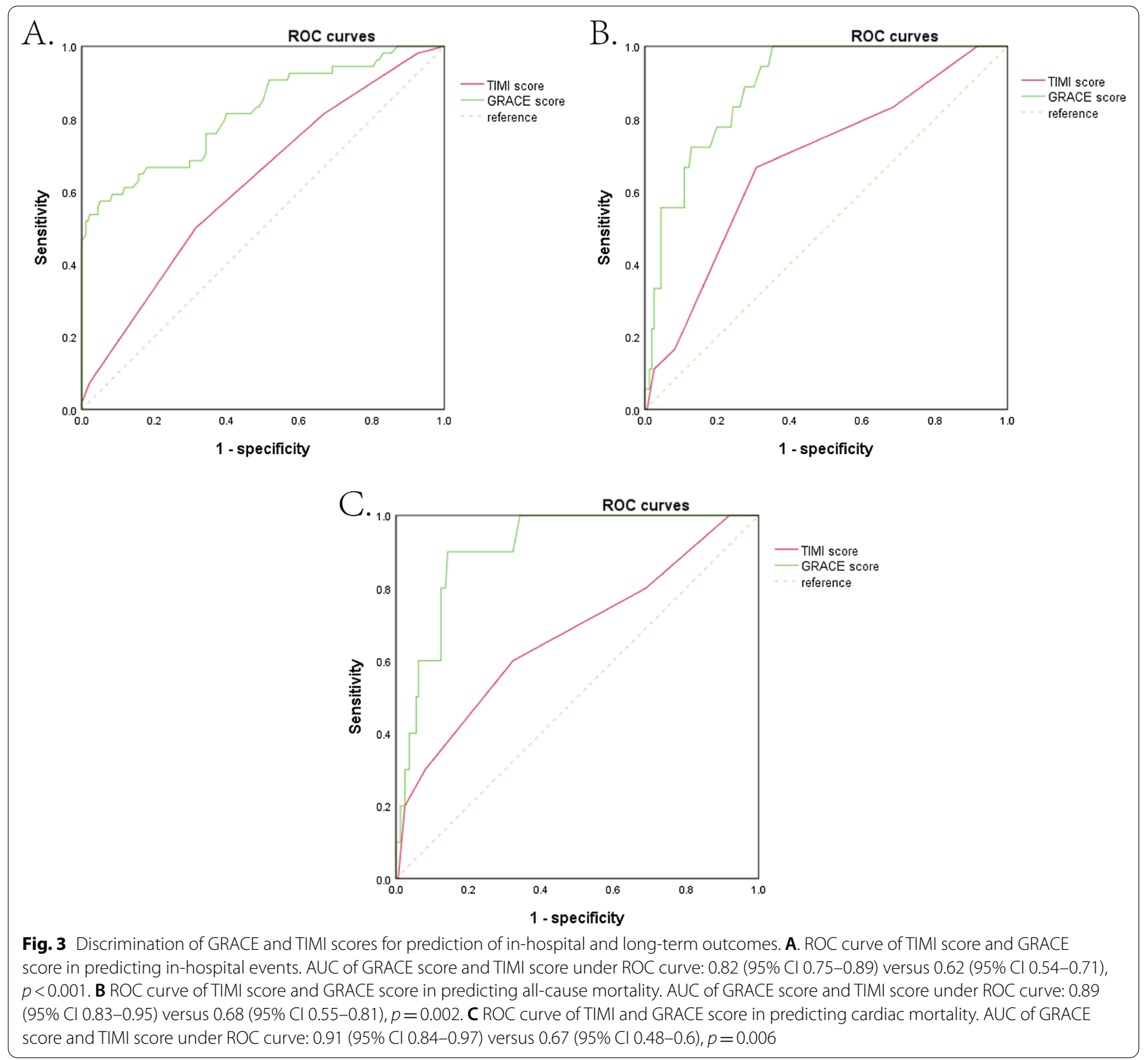

score among the East Asian NSTEMI population, our study focused on the NSTEMI patients instead of the ACS group, who most likely are in urgent need of aggressive invasive therapy upon risk stratification. As the first study, we examined the in-hospital and 4-year long-term outcomes' predictive value of these two major risk scores in current clinical practice.

The baseline characteristics of East Asian populations were largely different from the Caucasian population. Compared to the original GRACE cohort [19], our population was less likely to have a history of hyperlipidemia, angina, myocardial infarction, CABG, and heart failure but more likely to have ischemia, stroke, hypertension, diabetes, and renal insufficiency. We observed a significantly low incident of hyperlipidemia (5.2\% vs. $43.6 \%)$ in our population, which may be due to the difference in races and lifestyles between East Asians and Caucasians. Similarly, one recent large Korea registry of 27,852 acute myocardial infarction patients showed that the dyslipidemia rate in Korea was 9.5\%, compared with $53 \%$ in the Europe and America cohort [20]. Our study found that GRACE has higher discriminatory power in predicting in-hospital events and long-term outcomes than TIMI in East Asian NSTEMI patients. Such finding echoes previous studies examining these two scores' prediction ability among different ethnic NST-ACS/NSTEMI groups 


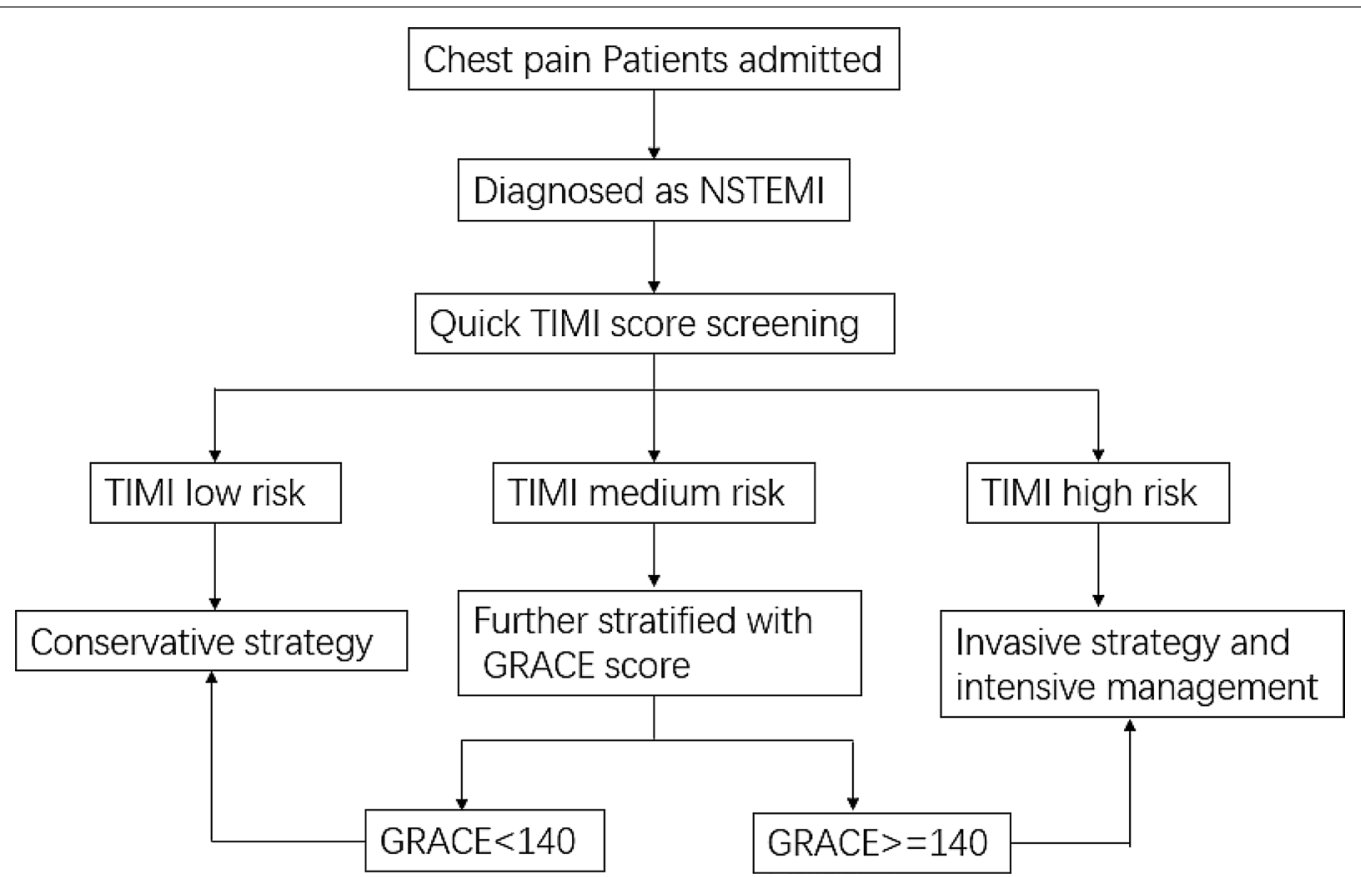

Fig. 4 Flow chat of screening high-risk patients with combination TIMI and GRACE risk score. NSTEMI non-ST-segment elevation myocardial infarction

Table 3 The risk heterogeneity of in-hospital events and longterm outcomes in TIMI medium risk group

\begin{tabular}{lllr}
\hline & GRACE high subgroup & $\begin{array}{l}\text { GRACE } \\
\text { non-high } \\
\text { subgroup }\end{array}$ & $p$ value \\
\hline In-hospital events & $30 / 76,39.5 \%$ & $6 / 66,9.1 \%$ & $<0.001$ \\
All-cause mortality & $12 / 54,22.2 \%$ & $0 / 51,0 \%$ & $<0.001$ \\
Cardiac mortality & $6 / 54,11.1 \%$ & $0 / 50,0 \%$ & 0.045 \\
\hline
\end{tabular}

Results are showed as value/n, percentage $(n \%)$

(such as UK, Portugal, and Latin-American population) [21-23]. A recent study on NSTEMI reported that the GRACE score predicted 28-day mortality better than the TIMI score (AUC: 0.87 vs. 0.54) [24]. Another study analyzed the 2184 NSTEMI patients in Korea, found that the AUC of ROC curves of GRACE and TIMI risk score is 0.750 versus 0.616 [25]. Until now, limited research studied the 4-year long-term outcomes between these two risk scores in the East Asian population. None of these studies tried to figure out the exact predictive discordance between TIMI and GRACE risk scores.

We found that GRACE stratified most patients into high-risk while TIMI stratified the majority as mediumrisk. Half of the TIMI medium group are GRACE high-risk patients with similar in-hospital events and long-term outcomes risk as TIMI high, mirroring the prior observed observations that TIMI tends to stratify high-risk patients into medium risk [13]. That may minimize doctors' attention to these patients. Previous studies in the Caucasian population were consistent with this result. A Britain study that examined 104 Non-ST-Elevation Acute Coronary Syndrome (NSTE-ACS) patients found that most patients were classified as a medium risk by TIMI score $(64 \%)$, yet classified as high risk with another risk stratification score-CMNW (Cheshire, Merseyside and North Wales Cardiac Network) score (60\%) [22]. Combining the TIMI and GRACE scores improves the predictive value, reserving the convenience of scoring while improving its diagnostic accuracy. To the best of our knowledge, the present study is the first to examine the discrepancy between risk scores in detail and explore the optimal risk stratification strategy among East Asian NSTEMI in both in-hospital and long-term outcomes.

Several findings can explain this discordance among risk scores. First, cardiac biomarker elevation only got 1 point in TIMI, which had a low weight in the TIMI scoring system but a high GRACE score. Therefore, although all our study population was troponin positive with a median GRACE score of 140 (135-145), approximately $30 \%$ of patients were still in TIMI low-risk groups $(\leq 2$ points). Second, the TIMI score variables did not comprehensively summarize high-risk patients' characteristics, such as heart rate, blood pressure, cardiac arrest, and ST-deviation, included in the GRACE score system. 
Therefore, the TIMI score underestimated patients' risk was particularly-risk patients. Those high-risk patients labeled as TIMI medium would be underestimated their risk of in-hospital events, delayed prompt invasive therapy, and subject to increase long-term mortality.

In conclusion, an optimal risk score requires the convenience of utility and the accuracy of discrimination. The GRACE risk score's main limitation is its apparent "complexity", requiring specific calculator tools for its use at the bedside. In contrast, TIMI was regarded as a risk score that is simpler to use and widely applicable in the emergency room than other risk scores [26]. Physicians may be reluctant to use risk scores at the bedside when they find it inconvenient and time-consuming. Moreover, most patients were decided with invasive therapy in the Emergency room. Thus, combine the two risk scores reserving the convenience of scoring while improving its diagnostic accuracy. The combination reaches a similar discriminative power as the GRACE score and preserves the TIMI score's easy use, meaning more patients can be easily scored in the emergency room, thereby improving patient care in routine clinical practice.

\section{Limitations}

The study was a retrospective study from Jan 1st, 2017, to Nov 1st, 2017. Although we collected the data consecutively, participants included in this study were from a single center that may lack representation which might cause recruitment deviation. Although their baseline characteristics did not differ from the published largest Chinese ACS patients' cohort [27], we could not eliminate any potential bias. Second, our study population is not as large as many registry studies. Although it is one of the largest NSTEMI East Asian population studies, our findings may not apply to patients who differ significantly from our population. Finally, 4 years after discharge, about $25 \%$ of patients lost to follow-up. Since our study was real-world data, the percentage of long-term follow-up in real-world populations was similar to previous studies $[28,29]$. Moreover, our studies compared the patients' characteristics between those with and without 4-year follow-up, showing no difference in patients' baseline characteristics and medical treatment. Therefore, the loss of follow-up should not skew our result very much.

\section{Conclusion}

In this retrospective study with East Asian NSTEMI patients, GRACE scores better-predicted patient's inhospital events and long-term outcomes than TIMI scores. Physicians can use the TIMI score for first screening and further categorize the medium-risk group by the
GRACE score, a novel way to facilitate risk score use and improve the identification of high-risk patients.

\section{Abbreviations}

NSTEMI: Non-ST segment elevation myocardial infarction; TIMI: Thrombolysis in myocardial infarction; GRACE: Global registry of acute coronary events; ACS: Acute coronary syndromes; STEMI: ST-segment elevation myocardial infarction; HR: Heart rate; SBP: Systolic blood pressure; Cr: Creatine; ROC: Receiver Operating Characteristic Curve; BNP: Brain natriuretic peptide.

\section{Supplementary Information}

The online version contains supplementary material available at https://doi. org/10.1186/s12872-021-02311-z.

Additional file 1. Table S1 showed the percentage of patients in different risk groups divided by TIMI and GRACE risk score. Kappa value showed the discordance of TIMI and GRACE risk score in grouping different-riskpatients. (Kappa value $<0.4$ implied that the correlation between TIMI and GRACE was poor.).

Additional file 2. Table S2 showed that after mixing medium- risk group and high-risk group in TIMI and GRACE risk groups, the incidence of in-hospital events and long-term outcomes showed no different. Results were showed as value (n).

Additional file 3. Table S3 showed the difference of baseline characteristics between patients with a high GRACE score and those with a low GRACE score in the TIMI medium-risk group. Results were showed as median (interquartile range), or percentage ( $n \%)$.

\section{Acknowledgements}

All the investigators thank the patients and clinicians included in this study. Data in this research were collected from Shanghai Renji Hospital, which deserves our gratefulness.

\section{Authors' contributions}

Y.L.: Dr. Lu had full access to all of the data in the study and takes responsibility for the integrity of the data and the accuracy of the data analysis. Dr. Lu contributed to the conception and design of the study, the data analysis, the data interpretation, the manuscript drafting, and the critical revision of the manuscript. L.S.: Dr. Shen contributed to the conception and design of the study, data interpretation, the manuscript drafting, and the critical revision of the manuscript. Dr. Shen contributed equally to the study. Y.M., L.S.: Ms. Miao and Dr. Shen contributed to the data interpretation, the manuscript drafting, and the critical revision of the manuscript. B.H.: Dr. Peterson contributed to the conception and design of the study, data interpretation, the manuscript drafting, and the critical revision of the manuscript.

\section{Funding}

This research was funded by the Clinical Research Center, Shanghai Jiao Tong University School of Medicine, Shanghai, China (Program Number: dly201512) to B. He, the National Natural Science Foundation of China to L. Shen (81900308) and the Information Development Project of Shanghai Municipal Commission of Economy and Informatization to C. Pan (202002009).

\section{Availability of data and materials}

The data set that supports the findings of this study are available from the corresponding author (drheben@126.com), upon reasonable request. The supplementary data is available online.

\section{Declarations}

\section{Ethics approval and consent to participate}

This study ethics has been approved by Shanghai Chest Hospital Ethics committee (ID: KS(Y) 1867). Written informed consent was waived for the retrospective study by Shanghai Chest Hospital Ethics committee. Our present 
medical research was conducted according to the principles expressed in the Declaration of Helsinki.

\section{Consent for publication}

Not applicable.

\section{Competing interests}

All the authors report no competing interests.

\section{Author details}

${ }^{1}$ Department of Cardiology, Shanghai Chest Hospital, Shanghai Jiao Tong University, Shanghai, China. ${ }^{2}$ Clinical Research Center, Shanghai Chest Hospital, Shanghai, China.

Received: 9 April 2021 Accepted: 5 October 2021

Published online: 07 January 2022

\section{References}

1. Benjamin EJ, Muntner P, Alonso A, et al. Heart disease and stroke statistics-2019 update: a report from the American heart association. Circulation. 2019;139(10):e56-528. https://doi.org/10.1161/cir.0000000000 000659.

2. Liu S, Li Y, Zeng X, et al. Burden of cardiovascular diseases in China, 1990-2016: findings from the 2016 global burden of disease study. JAMA Cardiol. 2019;4(4):342-52. https://doi.org/10.1001/jamacardio.2019.0295.

3. Du X, Patel A, Anderson CS, Dong J, Ma C. Epidemiology of cardiovascular disease in China and opportunities for improvement: JACC international. J Am Coll Cardiol. 2019;73(24):3135-47. https://doi.org/10.1016/j.jacc. 2019.04.036.

4. Iantorno M, Shlofmitz E, Rogers T, et al. Should non-ST-elevation myocardial infarction be treated like ST-elevation myocardial infarction with shorter door-to-balloon time? Am J Cardiol. 2020;125(2):165-8. https:// doi.org/10.1016/j.amjcard.2019.10.012.

5. Puelacher C, Gugala M, Adamson PD, et al. Incidence and outcomes of unstable angina compared with non-ST-elevation myocardial infarction. Heart (British Cardiac Society). 2019;105(18):1423-31. https://doi.org/10. 1136/heartjnl-2018-314305.

6. Puymirat E, Simon T, Cayla G, et al. Acute myocardial infarction: changes in patient characteristics, management, and 6-month outcomes over a period of 20 years in the FAST-MI program (French registry of acute ST-elevation or non-ST-elevation myocardial infarction) 1995 to 2015. Circulation. 2017;136(20):1908-19. https://doi.org/10.1161/circulationaha. 117.030798 .

7. Anderson JL, Morrow DA. Acute myocardial infarction. N Engl J Med. 2017;376(21):2053-64. https://doi.org/10.1056/NEJMra1606915.

8. Barnett R. Acute myocardial infarction. Lancet (London, England). 2019;393(10191):2580. https://doi.org/10.1016/s0140-6736(19)31419-9.

9. Roffi M, Patrono C, Collet JP, et al. 2015 ESC guidelines for the management of acute coronary syndromes in patients presenting without persistent ST-segment elevation: task force for the management of acute coronary syndromes in patients presenting without persistent STsegment elevation of the European Society of Cardiology (ESC). Eur Heart J. 2016;37(3):267-315. https://doi.org/10.1093/eurheartj/ehv320.

10. Antman EM, Cohen M, Bernink PJ, et al. The TIMI risk score for unstable angina/non-ST elevation Ml: a method for prognostication and therapeutic decision making. JAMA. 2000;284(7):835-42. https://doi.org/10.1001/ jama.284.7.835.

11. Gong IY, Goodman SG, Brieger D, et al. GRACE risk score: sex-based validity of in-hospital mortality prediction in Canadian patients with acute coronary syndrome. Int J Cardiol. 2017;244:24-9. https://doi.org/10. 1016/j.jijcard.2017.06.055.

12. Bueno H, Fernández-Avilés F. Use of risk scores in acute coronary syndromes. Heart (British Cardiac Society). 2012;98(2):162-8. https://doi.org/ 10.1136/heartjnl-2011-300129.

13. Yan AT, Yan RT, Tan M, et al. Risk scores for risk stratification in acute coronary syndromes: useful but simpler is not necessarily better. Eur Heart J. 2007;28(9):1072-8. https://doi.org/10.1093/eurheartj/ehm004.

14. Collet JP, Thiele H, Barbato E, et al. 2020 ESC guidelines for the management of acute coronary syndromes in patients presenting without persistent ST-segment elevation. Eur Heart J. 2021;42(14):1289-367. https://doi.org/10.1093/eurheartj/ehaa575.

15. Hall M, Bebb OJ, Dondo TB, et al. Guideline-indicated treatments and diagnostics, GRACE risk score, and survival for non-ST elevation myocardial infarction. Eur Heart J. 2018;39(42):3798-806. https://doi.org/10.1093/ eurheartj/ehy517.

16. Jiang $M, \mathrm{He} B$, Zhang $\mathrm{Q}$. Timing of early invasive intervention in patients with moderate to high risk acute coronary syndromes. J Interv Cardiol. 2012;25(1):10-8. https://doi.org/10.1111/j.1540-8183.2011.00685.x.

17. Kofoed KF, Kelbæk H, Hansen PR, et al. Early versus standard care invasive examination and treatment of patients with non-ST-segment elevation acute coronary syndrome. Circulation. 2018;138(24):2741-50. https://doi. org/10.1161/circulationaha.118.037152.

18. Koshizaka M, Lopes RD, Newby LK, et al. Obesity, diabetes, and acute coronary syndrome: differences between Asians and whites. Am J Med. 2017;130(10):1170-6. https://doi.org/10.1016/j.amjmed.2017.03.030.

19. Granger CB, Goldberg RJ, Dabbous O, et al. Predictors of hospital mortality in the global registry of acute coronary events. Arch Intern Med. 2003;163(19):2345-53. https://doi.org/10.1001/archinte.163.19.2345.

20. Sim DS, Jeong MH. Differences in the Korea acute myocardial infarction registry compared with western registries. Korean Circ J. 2017;47(6):81122. https://doi.org/10.4070/kcj.2017.0027.

21. de Araújo GP, Ferreira J, Aguiar C, Seabra-Gomes R. TIMI, PURSUIT, and GRACE risk scores: sustained prognostic value and interaction with revascularization in NSTE-ACS. Eur Heart J. 2005;26(9):865-72. https://doi.org/ 10.1093/eurheartj/ehi187.

22. Rawlings C, Oglesby K, Turner J, Sen A. Comparison of two clinical scoring systems in risk stratification of non-ST elevation acute coronary syndrome patients in predicting 30-day outcomes. Emerg Med J EMJ. 2012;29(1):40-2. https://doi.org/10.1136/emj.2010.102301.

23. Torralba F, Navarro A, la Hoz JC, et al. HEART, TIMI, and GRACE scores for prediction of 30-day major adverse cardiovascular events in the era of high-sensitivity troponin. Arq Bras Cardiol. 2020;114(5):795-802. https:// doi.org/10.36660/abc.20190206.

24. Chunawala ZS, Hall ME, Arora S, et al. Prognostic value of shock index in patients admitted with non-ST-segment elevation myocardial infarction: the ARIC study community surveillance. Eur Heart J Acute CardiovasC Care. 2021. https://doi.org/10.1093/ehjacc/zuab050.

25. Kim HK, Jeong MH, Ahn Y, et al. A new risk score system for the assessment of clinical outcomes in patients with non-ST-segment elevation myocardial infarction. Int J Cardiol. 2010;145(3):450-4. https://doi.org/10. 1016/j.ijcard.2009.06.001.

26. Poldervaart JM, Langedijk M, Backus BE, et al. Comparison of the GRACE, HEART and TIMI score to predict major adverse cardiac events in chest pain patients at the emergency department. Int J Cardiol. 2017;227:65661. https://doi.org/10.1016/j.ijcard.2016.10.080.

27. Chen YH, Huang SS, Lin SJ. TIMI and GRACE risk scores predict both shortterm and long-term outcomes in Chinese patients with acute myocardial infarction. Acta Cardiol Sin. 2018;34(1):4-12. https://doi.org/10.6515/acs. 201801_34(1).20170730b.

28. Danchin N, Farnier M, Zeller M, et al. Long-term outcomes after acute myocardial infarction in patients with familial hypercholesterolemia: the French registry of acute ST-elevation and non-ST-elevation myocardial infarction program. J Clin Lipidol. 2020;14(3):352-360.e356. https://doi. org/10.1016/j.jacl.2020.03.008.

29. Zdanyte M, Wrazidlo RW, Kaltenbach S, et al. Predicting 1-, 3- and 5-year outcomes in patients with coronary artery disease: a comparison of available risk assessment scores. Atherosclerosis. 2021;318:1-7. https://doi. org/10.1016/j.atherosclerosis.2020.12.007.

\section{Publisher's Note}

Springer Nature remains neutral with regard to jurisdictional claims in published maps and institutional affiliations. 